International Journal of Engineering \& Technology, 7 (4.19) (2018) 914-918
International Journal of Engineering \& Technology
SPC
Website: www.sciencepubco.com/index.php/IJET
Research paper

\title{
Locations Ranking using Page Rank Algorithm
}

\author{
Ghaidaa A. Al-Sultany*1, Asraa A. Abd Al-Ameer ${ }^{2}$ \\ ${ }^{1,2}$ Department of Information Network, Babylon University, Babel, Iraq \\ *Corresponding Author E-mail: ghaidaa.almulla@itnet.uobabylon.edu.iq
}

\begin{abstract}
Nowadays, large amount of user-generated data can obtained from social media (e.g., Instagram and Flicker) .People sharing their travel experiences with geo-tagged photo through these media, and the photo itself has important information like title, tags and location.Thesetypes of data provide a new perspective for us to understand the contexts of users. In this paper we focused on tourism service by collecting and analyzing geo-tagged photo from the social media to identify the most popular tourist places and rank them based on user location. We used PageRank algorithm that rank locations based on the relation between the locations and a set of tags that discovered by weighting scheme. Thus, an intelligent location-based tourist services can be provide to people. The work experiments have shown encouraging results in terms of ranking the given locations.
\end{abstract}

Keywords: Geo-tags resources, Social media data, Information ranking, Tourist attraction, Smart cultural tourism

\section{Introduction}

In the past few years, information and communication technology tools and applications (e.g., mobile devices) are widespread and many of smart applications have been diversely used[1].The benefits of social media and expansion abilities human exploration can boost by the mobile base and web service [2]. The use of social media is a growing phenomenon in contemporary society and its platform used as a means of communications and sharing information [3]. The users are capable to share media anywhere and at anytime to cooperative communicate and interacting with other users. They share their data like photos and videos and exchange their experiences with other users through it [2]. Using these data from social media can improve the utilization of it and provide a good personalized services to users[1].

The large amounts of data shared on social media being geotagged reflect activities and interests of people's, thus offering capabilities to analyze and evaluate our world. By associated geographic information, these social-media items able us to understand what geographic areas people are interest in it. Mining this dataset can provide greatly valuable to a several set of applications including improving health [4] ,city management [5] and more. The data of social media (e.g., Flicker, Twitter, Instagram) called geo-tagged when it is associated with geographic coordinates, usually expressing where the item was generated[6].

Basically, many types of information can be collect from social networking services.Therefore, social media has been considered as a source of tourist resources [7].Consequently, the behaviors of tourists can be observe through social media geo-tagged big data, thus providing backing for the applications such as intelligent travel recommendations and tourism resource development [8]. In this paper, we show an idea to exploit geo-tagged photos extracted from SNS(e.g., Flickr) to provide a service to people about the most attractive tourist places with respect to their location.
From defining a user's location the system can extract, collect the data (consisting of photos and tags ) that are linked to nearby that location and analyze them as an agile way for provide service to people that guiding and help them as much as possible in the time of their traveling.

The paper is organized as follows. In Section 2, introduces some studies related to the background of using geo-tagged resources to provide a specific services to people. Section 3 show the methodology of our work that consist of data representation, tags similarity and explain PageRank algorithm and how we used it to rank tourist locations to provide a tourist service to users. Section 4 show the results and experiment discussion of our work .Section 5 draws the conclusion of our study.

\section{Related Work}

Recently, there are many studies that benefit from the social network service resources such as geo-tagged photos which aim to provide a specific tourist service to users and help in the development of tourism. Where geo-tagged resources were used by the authors in [8],they proposed CFSFDP , a new clustering method in discovering thetourist attraction and they added zoning and standardization step in the process of spatial clustering to make their theory more flexible to the clustering scenes when there are large difference in regional density. In addition and to set the tag vector for each initial cluster, they used TF-IDF method and then merged adjacent semantically similar clusters by perform vector similarity calculation which enables more efficient in tourist attractiondiscovering that has an irregular shape and a large geographical area. Their experimental results displayed that their approach is much higher in the accuracy of the classification ,has better flexibility in the case of an irregular density distribution and enables distinguishing adjacent high-density areas. In [9] the authors showed an idea to exploit the tags attached on geographical photo that published by uses in SNS to help finding the most familiar locations for enjoy in specific food by using traditional dishes keyword. In order toused geo-tagged resources for ranking locations, they used LocHITS algorithm and 
modified the value of nodes with relevant locations and tags. Besides, they extend LocHITS to LocHITSS algorithm by using similarity between the set of tags of each location and the set of common tags. Furthermore, in order to calculate the weight of tags and apply them into the LocHITSTF algorithm, they used term frequency of tags in each location. The experiments showed that the GeoHITS algorithm converge quickly, and the results had high ranking values.

As another approach other authors focused more on practical and structural forms of Flickr mining rather than algorithmic and technical forms of it such as in [10],the researcher proposes a framework that used geo-tagged photos in Queensland, Australia ( attractive tourist destination ) to extract associative points-ofinterest patterns. The framework consist of two popular data mining techniques: making for the points-of-interest detection a clustering, and association rules mining for associative points-ofinterest patterns. While the authors in [11]find the tourist attractions and places from the geo-tagged photos that have geographical location information and extracted representative photos and corresponding text labels, able the users to use text or pictures to search tourist attractions places.

\section{Methodology}

In our work we focus on defining the problems and fundamental issues that may face when want to provide to tourists some helpful information associated to their trip, by using geo-tagged social media data we can identify tourist places from user's position and rank them. To ranking locations, we depend on the relationships between tags and locations. The problem of ranking location focus on data that can obtained from SNS by using tags that attached with it determine the features of locations. Where a tag $\left(t_{i}\right)$ is a label attached to photo in order to identify or describe the its contexts, location $\left(l_{j}\right)$ is a name of a country, city, or a place that used for ranking and it is identified by a collection of tags over social media. The relation defines the link between a tag $\left(t_{i}\right)$ and a location $\left(l_{j}\right)$, so if the tag $\left(t_{i}\right)$ is contained in location $\left(l_{j}\right)$ or in other words, if location $\left(l_{j}\right)$ has been tagged with tag $\left(t_{i}\right)$, this means that there is a relation (link) between the tag and the location.

We use a collection of geo-tagged photos from SNS and applying ranking method in order to identify and find the better location for the user. The tags that appear on many locations should be chosen for using in this work. Besides, we inspired from [1]that to find the best location for the user by ranking method, we use a collection of tags that occurs in many locations. Because there is no meaning for some tags with the other location that did not contain them. We need to use a collection of common tags. The common tags are defined as :

$$
\mathrm{T}^{\mathrm{c}}=\left\{t_{i}\left|t_{i} \in \mathrm{T},\right| \mathrm{L}^{t_{i}} \mid \geq \propto\right\}
$$

Where $\mathrm{t}$ is a tag, $l$ is a location, T, is a collection of tags, Lis a collection of locationsand $\mathrm{L}^{t}$ is a collection of locations contain tag t.

In social media, there are large scale use for tags from which it can be possible to apply a process to select the most popular tags that has relationship closed to the keyword.The workflow of our STS ( Social Tourist Service) is shown in the Figure1.

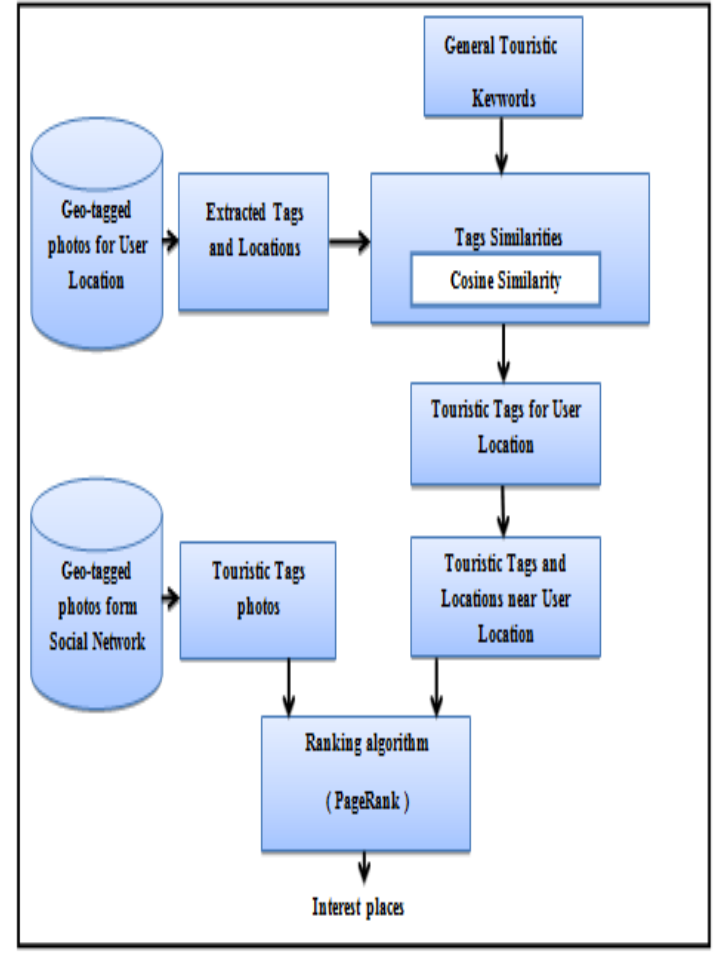

Fig. 1:The proposed research diagram

\subsection{Data Representation}

Our system used dataset taken from [12]. This dataset crawled from Flickr and it consists of a sample of geo-tagged photos with the corresponding file metadatathat consist of image metadata in CSV format that contain information's fieldsabout all images.Each field define a specific information on the image. 5000 geo-tagged photos have been taken from the dataset for testing this work. The selected dataset has been apart into two parts, the first one has contained a set of locations that taken from photos with its tags collected based on their closeness to user location or given area. The latitude and longitude of the location where the photo was taken using Google map API service have been utilized to get the locations that near the user or given area. The second part of the dataset collected based on using a keyword related to tourism through calculating the cosine similarity [13] between set of most common tourist keywords and the tags of all photos that contain a set of tourist tags.

\subsection{Tags Similarity}

In the process of ranking, we use tourist tags to decision a list of ranking tourist locations and that has been done by PageRank algorithm .So, to get a list of tourist tags, we calculate the cosine similarity measure between each tag for each location with the general tourist keywords to get the tourist tags only.

After selecting a collection of tourist tags, we calculated the number of locations that contain the tourist tag to get a collection of common tags that occurred in many locations, and that for depend on the tags of locations to compute the ranking value for each location. Thus ,that will change the weight of vertices (tags and locations) and make the result of ranking by PageRank more accurate.

Each location will be affected by its tagsand vice versa; each tag will be affected by its location. Thus, we need to compute the weight of tags in each location; and we inspired that from [1] where the number of occurrence for each tourist tag in the location will count and will compare with the total number of tags in that location.It's calculated as follows :

$w_{i j}=\frac{p(t i, l j)}{\max \left\{p\left(t_{k}, l j\right) \mid t_{k} \in T^{l j}\right\}}$ 
With $p\left(t_{i}, l_{j}\right)$ is the count of occurrence of tag $t_{i}$ in location $l_{j}$ and the maximum occurrence of $t_{k}$ ( $t_{k}$ is a tag in the collection of location $l_{j}$ tags).In our work, we used the relationships between tags and locations such that value of each tags in each location and value of each location that contain the tag.

\subsection{Formalization of Location Ranking}

PageRank algorithm is proposed by Sergey Brin and Lawrance Page , it's one of the most commonly ranking algorithm that used for ranking the various pages and its currently used by Google search engine[14].

From studying results of[15], we have proposed the PageRank algorithm to find the best location, in which each node is definedby a tag or a location. The grade of a vertex of a graph in graph approach, is the count of edges that incident to the vertex.In directed-graph, there are two kinds of degree of vertex p: Indegree " the count of links that points to vertex p" and Out-degree "the count of links from p point to other points".Differently, the count of In-degree and Out-degree of vertexin an undirected graph is equal.

In this paper and for ranking locations, we depend on the relation between tags and locations and an undirected graph we used to represent data $\mathrm{G}=\langle\mathrm{N}, \mathrm{L}\rangle$ where $\mathrm{N}$ represented the collection of nodes corresponds to a tag $t_{i}$ or a location $l_{j}$ and $\mathrm{L}$ is the collection of edges $\left(t_{i}, l_{j}\right) \in \mathrm{L}$ indicates location $l_{j}$ contains tag $t_{i}$. To calculate values for nodes, we found that we should chose the tags that occur in various locations because if only one tag show in a location for just a few times then it has barren for another locationsranking.

At the beginning and to get the most interested locations, the initial approximation of PageRank for locations $(\forall v \in \mathrm{N})$ would begin with an estimated PageRank of $1 / n$ ( $n$ is the number of location in the place) and the PageRank of tags would begin with an estimated PageRank of $1 / \mathrm{m}$ ( $\mathrm{m}$ is the number of tourist tags) as follows :

$\operatorname{PR}\left(l_{j}\right)=\frac{1}{n}$

$\operatorname{PR}\left(t_{i}\right)=\frac{1}{m}$

Then, for each iterations the PageRank of all locations will depend on the PageRank of tourist tags that it contained and the probability of transitioning from tag to location and that will calculatedby the formulas as follows :

$\operatorname{PR}\left(l_{j}\right)=\sum_{i=1}^{m} P R\left(t_{i}, l_{j}\right) \cdot p_{i j}$

In addition to that , for each iterations the PageRank of all tags will depend on the PageRank of location that contained in it and the probability of transitioning from location to tag and that will computed by the formulas as follows :

$\operatorname{PR}\left(t_{i}\right)=\sum_{j=1}^{n} P R\left(l_{j}, t_{i}\right) \cdot p_{j i}$

Because the edges in the graph that we deal with it in our work may have weight $\left(p_{i j}\right)$, the probability of transitioning from tag to $\operatorname{location}\left(p_{i j}\right)$ and the probability of transitioning from location to $\operatorname{tag}\left(p_{j i}\right)$ is defined in these cases as follow [16]:

$p_{i j}=\frac{w_{i j}}{\sum_{j \in O u t(i)} w_{i j}}$

$p_{j i}=\frac{w_{j i}}{\sum_{i \in O u t(j)} w_{j i}}$

In this study we used undirected graph to rank location based of its tags .So, the weight of edges between the tag and location and vice versa is the same. At the end, the final PageRank of tags and locations will calculate as follows:
$\operatorname{PR}\left(t_{i}\right)=\frac{d}{m}+(1-d) \cdot \sum_{j \in \operatorname{In}(i)} \operatorname{PR}\left(l_{j}\right) \cdot p_{j i}$

$\operatorname{PR}\left(l_{j}\right)=\frac{d}{n}+(1-d) \cdot \sum_{i \in \operatorname{In}(j)} \operatorname{PR}\left(t_{i}\right) \cdot p_{i j}$

Where $\mathrm{n}$ is the total number of locations, $\mathrm{m}$ is the total number of tags and $\mathrm{d}$ is a damping factor which can be set to between 0 and $1, \mathrm{~d}=0.85$ in this paper.

\section{Result and Experimental Discussion}

In this section, we introduced the dataset that collected from SNS[12] and used in our work and shows implementation steps to rank locations based on tags of them and its results.

\subsection{Dataset}

To perform our work with real-world data, a data of geo-tagged photos on a world-wide scale is presented. The data contains a sample of 5000 geo-tagged photos posted and shared by many users on over the world and crawled from Flickr with the corresponding metadata like title ,tags, latitude, longitude ,date taken ,date uploaded and other attached featuresbelong to that photos.

\subsection{Experimental Results}

In our work ,we used two dataset, the first for the locations around the given area, we assume that the user is in a New Orleans city which is one of Louisiana state in America country, using the position of the city ( latitude and longitude ) the application collected a set of locations found in it by using one of Google map API services which is Geocoding API service, the locations that collect its depend on latitude and longitude metadata of photos that collect from Flicker and its considered dataset 1 . The second for tourist words ( tags ) extracted from geotagged photos that posted and shared by many users on over the world when they want to tags or comment through Flicker and that by using keyword related to tourism and its considered dataset 2.

Two different cases were experienced to test the results of our work before working on the 5000 geo-tagged photos, in each case there is a different number of tourist locations and non-tourist locations. . A 500 different photos ( tourist and non-tourist photos) were taken from different places around the world, 31 photos from New Orleans city which we want to find the tourist location in it and the other from different locations around the worlds. The 31 photos were captured and shared from 20 different location in New Orleans.In order to evaluated the results, two measures precision and recall have been used and in the F-measure the two measures are used together to provide one measurement for the evaluation of our system as show below [1]:

$$
\begin{aligned}
& p=\frac{\mid \text { \{reler }}{r=} \\
& F-\text { measu }
\end{aligned}
$$

Case 1 : In this case , 31 different photos fromNew Orleans city that captured and share from 20 different location have been used. From the 20 locations , 17 of them its tourist location and the rest (3 locations ) it's not. The result gave the 17 tourist locations and two non-tourist location as shown in Table 1 based on tags relevant to tourism that collect from social network that shown in Table 2. 
Table 1: The rank of tourist places

\begin{tabular}{|l|l|l|}
\hline The Locations & Locations Rank Value & $\begin{array}{l}\text { Touristic/Not } \\
\text { Touristic }\end{array}$ \\
\hline 1725 St Roch Ave & 0.06374373294351214 & Touristic \\
\hline 343 Carondelet St & 0.05903317144347555 & Touristic \\
\hline 425 Basin St & 0.05804262640382418 & Touristic \\
\hline 5100 Canal Blvd & 0.05780526628352904 & Touristic \\
\hline 2900 Loyola Ave & 0.056617381462497396 & Touristic \\
\hline 102 City Park Ave & 0.05542947975743634 & Touristic \\
\hline 401 St Peter St & 0.05428507363539069 & Touristic \\
\hline 1 Canal St & 0.051934072891302155 & Touristic \\
\hline 733 Bourbon St & 0.05189291420918382 & Touristic \\
\hline 317 N Rampart St & 0.051882888048774986 & Not Touristic \\
\hline 800 Decatur St & 0.050292397660818715 & Not Touristic \\
\hline 698 Loyola Ave & 0.049554242436851303 & Touristic \\
\hline Aquarium Dr & 0.049551278442396325 & Touristic \\
\hline Collins Diboll Cir & 0.04952696167338881 & Touristic \\
\hline 90057 Diagonal Dr & 0.04951506158874967 & Touristic \\
\hline 2604 Coliseum St & 0.04950188575578165 & Touristic \\
\hline 5016 Willow St & 0.047148192963958856 & Touristic \\
\hline Mardi Gras World & 0.047122627228623294 & Touristic \\
\hline 1320 Mardi Gras Blvd & 0.047120745170504824 & Touristic \\
\hline
\end{tabular}

Table 2: Tourist tags (Dataset 2) Tourist Tags

cemetery - grave - river - trip -vault - water - tourist - photo - travel trees - mississippiriver - garden - tour - tourism

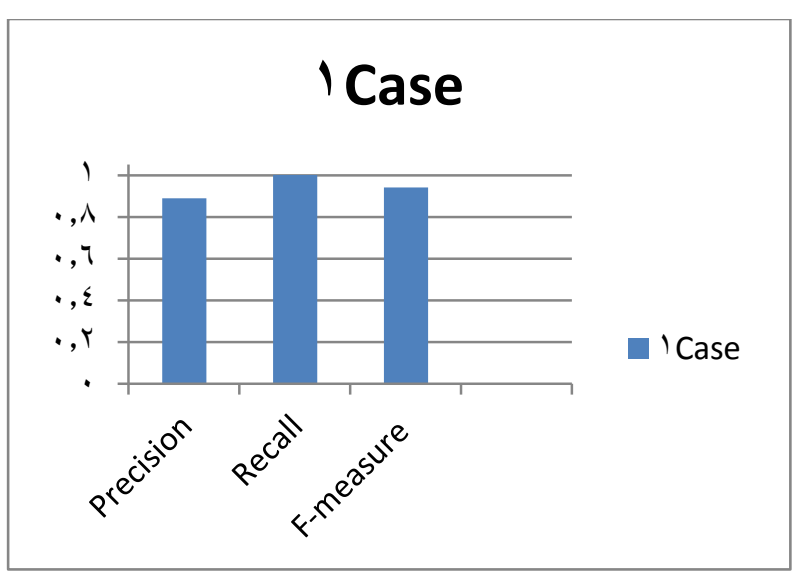

Fig. 2:Performance measure for case 1

Case 2: In this case, 31 different photos fromNew Orleans city that captured and share from 20 locationhave been used. From the 20 locations , 14 of them its tourist location and the rest (6 locations ) it's not. The result gave the 14 tourist locations and four nontourist location as shown in Table 3 based on tags relevant to tourism that collect from social network that shown in Table 4.

Table 3: The rank of tourist places

\begin{tabular}{|c|c|c|}
\hline The Locations & Locations Rank Value & $\begin{array}{c}\text { Touristic/Not } \\
\text { Touristic }\end{array}$ \\
\hline 1725 St Roch Ave & 0.0693876101165103 & Touristic \\
\hline 425 Basin St & 0.06273799374822389 & Touristic \\
\hline 5100 Canal Blvd & 0.06246092639954528 & Touristic \\
\hline 2900 Loyola Ave & 0.06107558965615227 & Touristic \\
\hline 102 City Park Ave & 0.05969025291275927 & Touristic \\
\hline 1 Canal St & 0.0564300352573853 & Touristic \\
\hline 401 St Peter St & 0.05642870260543905 & Touristic \\
\hline 2604 Coliseum St & 0.05374396135265701 & Touristic \\
\hline 800 Decatur St & 0.05374396135265701 & Not Touristic \\
\hline Collins Diboll Cir & 0.05336007673913605 & Touristic \\
\hline 90057 Diagonal Dr & 0.0533595047898433 & Touristic \\
\hline 733 Bourbon St & 0.05335942624967543 & Touristic \\
\hline 5016 Willow St & 0.050292384690543056 & Not Touristic \\
\hline 1320 Mardi Gras Blvd & 0.05029098310627568 & Touristic \\
\hline Mardi Gras World & 0.05029075514396734 & Touristic \\
\hline 433 Bolivar St & 0.04999289570900823 & Not Touristic \\
\hline 8025 St Charles Ave & 0.04999289570900823 & Not Touristic \\
\hline
\end{tabular}

Table 4: Tourist tags (Dataset 2) Tourist Tags

cemetery - grave - tomb - flower- photo - vault - water - tourist - travel trip - mississippiriver - garden - tour - tourism

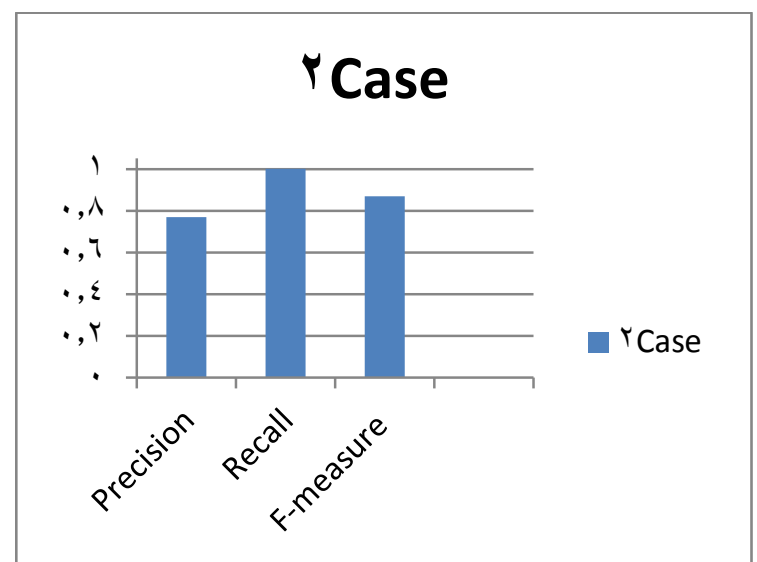

Fig. 3: Performance measure for case 2

Besides, our system has been implement on datacontains a sample of 5000 geo-tagged photos posted and shared by many users on over the world and crawled from Flickr. The first dataset is contain a set of locations that near user location, we assume that the user is in New Orleans city which is on of Louisiana state in America country. The first dataset described in Table 5.

Table 5: Dataset1 ( The names of locations in New Orleans city with its tags)

\begin{tabular}{|c|c|}
\hline $\begin{array}{l}\text { Location } \\
\text { name }\end{array}$ & Location tags \\
\hline $\begin{array}{l}2900-2998 \\
\text { Loyola Ave }\end{array}$ & $\begin{array}{l}\text { cemetery- grave- louisiana -neworleans -vault- crypt - } \\
\text { fuchs }\end{array}$ \\
\hline $\begin{array}{l}102 \text { City Park } \\
\text { Ave }\end{array}$ & $\begin{array}{l}\text { door - cemetery - rust - Louisiana- neworleans - chain } \\
\text { doorknob - padlock - crypt - forlorn - grave - warning - } \\
\text { tomb - gravesite }\end{array}$ \\
\hline $\begin{array}{ll}5100 & \text { Canal } \\
\text { Blvd } & \end{array}$ & $\begin{array}{l}\text { flowers - cemetery - grave - gold - golden - louisiana - } \\
\text { paint - neworleans - vault -bouquet - crypt - grave } \\
\text { headstone - tomb -brotherhood - boilermakers - } \\
\text { shipbuilders }\end{array}$ \\
\hline 425 Basin $\mathrm{St}$ & $\begin{array}{l}\text { flowers - blue - roses - strange - grave - Louisiana - } \\
\text { purple neworleans - eerie - vault - crypt - cemetery - } \\
\text { cult vault - priestess - voodoo - marieleveau - ochre - } \\
\text { soldier - memorial - revolution - revolutionarywar - } \\
\text { black - dark - magic - occult - } \\
\text { sinistergravedeathcryptneworleanslouisianaeeriemacabre } \\
\text { - death - macabre }\end{array}$ \\
\hline $\begin{array}{l}1725 \text { St Roch } \\
\text { Ave }\end{array}$ & $\begin{array}{l}\text { strange - grave - death - louisiana - god - neworleans - } \\
\text { cemetary - religion - jesus - eerie - graves - macabre - } \\
\text { hdr - gravesite - bigeasy - stroch - camposanto }\end{array}$ \\
\hline $\begin{array}{ll}1914 & \text { Tea } \\
\text { Room Dr } & \end{array}$ & $\begin{array}{l}\text { bird - nature - animals - zoo - louisiana - wildlife - } \\
\text { neworleans - parakeet - audubonzoo- orangutan - } \\
\text { monkey - primate - feline - jaguar - growl - cat - } \\
\text { aligator - bayou - albino - gator - swamp - tropical - red } \\
\text { - colorful - toucan }\end{array}$ \\
\hline
\end{tabular}

The second dataset is contain a common tourist tags that used by many users around the world when they published geo-tagged photos in Flicker as described in Table 6.

Table 6: Dataset 2 ( Tourist tags with the number of occurrence)

\begin{tabular}{|l|l|l|l|}
\hline Tourist tags & $\begin{array}{l}\text { Number of } \\
\text { locations } \\
\text { that contain } \\
\text { it }\end{array}$ & Tourist tags & $\begin{array}{l}\text { Number of } \\
\text { locations that } \\
\text { contain it }\end{array}$ \\
\hline nature & 110 & bird & 34 \\
\hline landscape & 78 & mountain & 33 \\
\hline sea & 68 & beach & 30 \\
\hline blue & 54 & bridge & 30 \\
\hline architecture & 50 & cemetery & 29 \\
\hline scenery & 48 & animals & 29 \\
\hline
\end{tabular}




\begin{tabular}{|l|l|l|l|}
\hline wood & 46 & louisiana & 28 \\
\hline red & 44 & flowers & 27 \\
\hline
\end{tabular}

The data that result from dataset 1 and dataset 2 have been taken to get the essentialtags for ranking the locations that belong to tourism. The locations are tagged by the people when they have been visiting it. So, all locations is described by a collection of tags.To filter data in each location, the tags that are related to tourist will be used.So, from the two dataset we noticed that each location have a set of tourist tags as shown in Table 7.

Table 7: The locations with its tourist tags

\begin{tabular}{|l|l|}
\hline Location name & Tourist tags \\
\hline $\begin{array}{l}\text { 2900-2998 Loyola } \\
\text { Ave }\end{array}$ & cemetery- louisiana \\
\hline 102 City Park Ave & cemetery - louisiana \\
\hline 5100 Canal Blvd & flowers - cemetery - louisiana \\
\hline 425 Basin St & flowers - blue - louisiana \\
\hline 1725 St Roch Ave & cemetery - louisiana \\
\hline 1914 Tea Room Dr & bird - nature - animals - louisiana \\
\hline
\end{tabular}

The rank of tourist places depend on user's favorites by tagging the photos that they share on social media. By the relation between the locations and tourist tags, we use the data in dataset2 (Table 6) to get the term frequency of the tourist tag in all locations of dataset 1 (Table 5). Undirected graph used to represent data where all vertex represent a location or a tag, and all edge represent the relationship between them. Here, we only taking into consideration the relationship tag-place or place-tag , without taking the relationship tag-tag or place-place. By applying PageRank algorithm depending on the relation between tags and locations we will get the most attractive tourist places and tourist tags in New Orleans city. Page rank algorithm will remove the locations that not contain any tourist tag and remove the tags that don't found in any location, So that will give a more accurate result as shown in Table 8 .

Table 8: The rank of tourist places

\begin{tabular}{|l|r|}
\multicolumn{2}{|c|}{ Table 8: The rank of tourist places } \\
\hline Rank of locations & PageRank value for the locations \\
\hline 1725 St Roch Ave & 0.18164971507234723 \\
\hline 1914 Tea Room Dr & 0.16600514487352738 \\
\hline 102 City Park Ave & 0.16451425775244655 \\
\hline 425 Basin St & 0.16451424071901233 \\
\hline 5100 Canal Blvd & 0.1616583215129468 \\
\hline 2900 Loyola Ave & 0.16165832006971925 \\
\hline
\end{tabular}

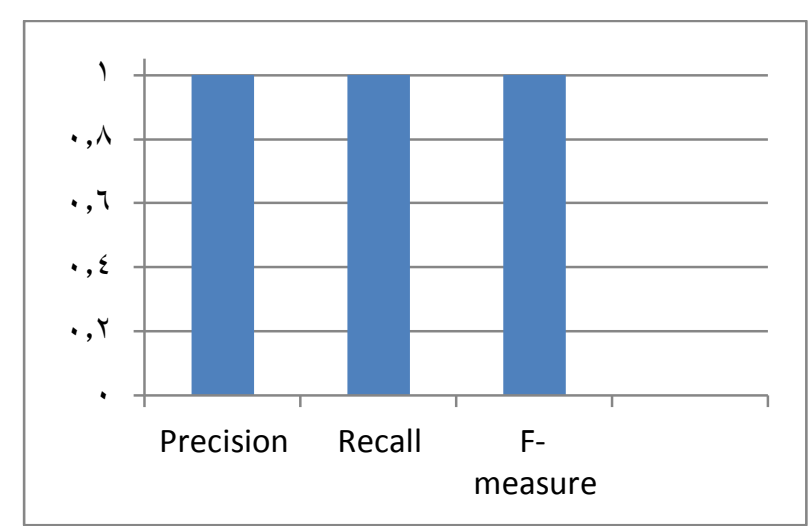

\section{Conclusion}

Fig. 4: Performance measure for the system

In this paper,a benefit has been shown from social network data. By given a particular geolocation,tourists can be provided with the most attractive tourist places in their area based on geo-tagged resources in social media. To ranking locations based on geotagged resources, we proposed PageRank algorithm and modified the values of the locations based on the tourist tags that contained it.By that,impressive information can find for tourists and providing effective information to the agents of travelling and that helping in the development of tourism.

\section{References}

[1] T. T. Nguyen, D. Camacho, and J. E. Jung, "Identifying and ranking cultural heritage resources on geotagged social media for smart cultural tourism services," Pers. Ubiquitous Comput., vol. 21, no. 2, pp. 267-279, 2017.

[2] I. Memon, L. Chen, A. Majid, M. Lv, I. Hussain, and G. Chen, "Travel Recommendation Using Geo-tagged Photos in Social Media for Tourist," Wirel. Pers. Commun., vol. 80, no. 4, pp. 1347-1362, 2015.

[3] L. Townsend and C. Wallace, "Social Media Research: A Guide to Ethics," Univ. Aberdeen, pp. 1-16, 2016.

[4] A. Sadilek, H. Kautz, and V. Silenzio, "Modeling Spread of Disease from Social Interactions," Proc. Sixth Int. AAAI Conf. Weblogs Soc. Media, pp. 322-329, 2012.

[5] C. Tech, A. Krebs, C. Tech, R. Schwartz, A. Langdon, and C. Tech, "CityBeat: Real-time Social Media Visualization of Hyper-local City Data," pp. 167-170, 2014.

[6] D. Flatow, M. Naaman, K. E. Xie, Y. Volkovich, and Y. Kanza, "On the Accuracy of Hyper-local Geotagging of Social Media Content," Proc. Eighth ACM Int. Conf. Web Search Data Min. WSDM' '15, pp. 127-136, 2015.

[7] J. J. Nguyen TT, Hwang D, Intelligent Distributed Computing III, vol. 237. Madrid, Spain, 2009.

[8] X. Peng and Z. Huang, "A Novel Popular Tourist Attraction Discovering Approach Based on Geo-Tagged Social Media Big Data," ISPRS Int. J. Geo-Information, vol. 6, no. 7, p. 216, 2017.

[9] T. Tri Nguyen and J. J. Jung, "Exploiting geotagged resources to spatial ranking by extending HITS algorithm," Comput. Sci. Inf. Syst., vol. 12, no. 1, pp. 185-201, 2015.

[10] I. Lee, G. Cai, and K. Lee, "Exploration of geo-tagged photos through data mining approaches," Expert Syst. Appl., vol. 41, no. 2, pp. 397-405, 2014.

[11] Y. Gao, J. Tang, R. Hong, Q. Dai, T.-S. Chua, and R. Jain, "W2Go," Proc. Int. Conf. Multimed. - MM '10, p. 123, 2010.

[12] H. Mousselly-Sergieh, D. Watzinger, B. Huber, M. Döller, E. Egyed-Zsigmond, and H. Kosch, "World-wide scale geotagged image dataset for automatic image annotation and reverse geotagging," Proc. 5th ACM Multimed. Syst. Conf. - MMSys '14, pp. 47-52, 2014.

[13] N. Pradhan, M. Gyanchandani, and R. Wadhvani, "A Review on Text Similarity Technique used in IR and its Application," Int. J. Comput. Appl., vol. 120, no. 9, pp. 29-34, 2015.

[14] N. V Pardakhe and R. R. Keole, "Analysis of Various Web Page Ranking Algorithms in Web Structure Mining," Int. J. Adv. Res. Comput. Commun. Eng., vol. 2, no. 12, pp. 4798-4803, 2013.

[15] M. Batra, S. Sharma, A. Prof, D. Of, C. Applications, and M. Rachna, "Comparative Study of Page Rank Algorithm With Different Ranking Algorithms Adopted By Search Engine For Website Ranking," Int. J. Comput. Technol. Appl., vol. 4, no. 1, pp. 8-18, 2013.

[16] C. C. Aggarwal, Recommender Systems. 2016. 